\title{
SCANINING ELECTRON MICROSCOPIC EVALUATION OF CHITOSAN NANOHYDROXYAPATITE SCAFFOLD FOR SOCKET HEALING IN RABBITS WITH INDUCED OSTEOPOROSIS
}

\author{
Hagar S. Gharib $M S c^{1^{*}}$, Afaf A. El-Sawa $P h D^{2}$, Sahar S. Karam $P h D^{2}$, Sara A. Hamza $P h D^{3}$
}

\begin{abstract}
INTRODUCTION: Significant development has been achieved with bioceramics and biopolymer scaffolds in the construction of artificial bone. In the present study, Chitosan- nanohydroxyapatite (CS/nHA) scaffolds have been developed as bone graft substitutes in rabbits with induced osteoporosis.

OBJECTIVES: To evaluate the effect of Chitosan- nanohydroxyapatite (CS/nHA) scaffold in bone tissue regeneration of extracted socket in rabbits with induced osteoporosis; using Scanning Electron Microscope and Energy Dispersive x-ray analysis [EDXA].

MATERIALS AND METHODS: Twenty NewZealand white female rabbits were randomly divided into 2 equal groups; group A (osteoporosis) with no graft material, and group B (osteoporosis with CS/nHA). After the experimental period the animals were sacrificed at 2 \& 4 weeks. The effect of CS/Nha was evaluated using Scanning Electron Microscope and Energy Dispersed x-ray analysis [EDXA]. The data obtained was tabulated and subjected to statistical analysis.

RESULTS: Scanning Electron Microscopic results revealed more trabecular bone thickness with regular bone surface, and uniform osteocyte lacunae in the group $\mathrm{B}$ (osteoporosis with CS/nHA) than group A (osteoporosis), and these results were proved by EDXA which showed more $\mathrm{Ca}$ and $\mathrm{P}$ ratio in the group $\mathrm{B}$ than group $\mathrm{A}$.

CONCLUSIONS: CS/nHA scaffold is considered an effective hard tissue engineering material with sufficient interconnected porosity and mechanical strength to allow cell adhesion, migration, growth and proliferation resulting in good integration with surrounding tissues, and rapid bone healing, thus it might be used in osteoporotic condition.
\end{abstract}

KEYWORDS: Osteoporosis, Extraction, Chitosan, Nanohydroxyapatite, Scaffold, Bone.

1- Assistant Lecturer, Oral Biology, Faculty of Dentistry, Alexandria University

2- Professor of Oral Biology, Faculty of Dentistry, Alexandria University

3- Lecturer of Oral Biology, Faculty of Dentistry, Alexandria University

*Corresponding author:

E-mail: hagarsamirgharib27@yahoo.co.uk

\section{INTRODUCTION}

Nanotechnology is the manipulation of molecular-sized materials to create new products and processes. It deals with fields such as physics, chemistry and biochemistry, and molecular biology (1).

Nanotechnology is a rapidly progressing field; it already has a great impact in orthopedics since it provides the ability to better mimic the native nano-environment of natural tissues (2). Nanomaterials (that is, materials with one fundamental dimension less than $100 \mathrm{~nm}$ ) can reduce implant infection, inhibit inflammation, and increase bone growth all without using pharmaceutical agents (3).

Bone tissue engineering is a specific area in nanotechnology where the development of nanostructured biomaterials may be able to replace hard and soft skeletal tissue, and biocompatible materials for tissue genesis. Other related areas also include creation of nanoporous biocapsules for cellular therapy. Tissue engineering stands to benefit most from the nanotechnology because of the growing ability to fabricate complex nanostructured materials (4).

The architecture of biomaterial scaffolds is crucial to the performance of the scaffold. Scaffolds not only provide a structural support for cell integration, but also regulate cell proliferation, differentiation and migration to form functional tissues (5).

Scaffolds for bone regeneration should meet certain criteria to serve this function, including mechanical properties similar to those of the bone repair site, biocompatibility and biodegradability at a rate commensurate with remodeling. Scaffolds serve primarily as osteoconductive moieties, since new bone is deposited by creeping substitution from adjacent living bone (6).

Based on the natural structure of bones, Chitosan/nanohydroxyapatite composite was prepared in this study. Nanohydroxyapatite (nHA) can be made either synthetically $(7,8)$ or isolated from natural sources $(9)$. However, instead of natural HA, the composite of chitosan with synthetic HA is more widely used as a bone graft substitute (10).

Hydroxyapatite (HA), is a major inorganic component of bone, that has been studied as a possible substitute material for hard tissue due to its high biocompatibility and osteoconductivity, and it has been used clinically as artificial bone and for dental implants substitute (11). However, the clinical use of HA as a bone substitute has 
been proved problematic due to its brittleness, and difficult to prevent the dispersion of HA granules and mold the granules into the desired shape (12).

HA nanoparticles means HA that have a size of $<100$ $\mathrm{nm}$ in diameter of width or length. They are used in various medical applications such as the delivery of antibiotics, growth factors, anticancer drugs, enzymes, and antigens for slow-release vaccinations. HA nanoparticles are known to have good bioactivity, high biocompatibility, and a flexible structure, which are necessary qualities in biomedicine. The effect of nanosized HA particles on the host tissue response have been encouraging, which has made the development of nanophase HA increasingly imperative (13).

Chitosan is a natural biopolymer consisting of $\beta(1 \rightarrow$ 4)-2-acetamido-d-glucose and $\beta(1 \rightarrow 4)$-2-amino-dglucose unit linkage (14) and a promising biomaterial for bone tissue engineering. In addition, chitosan can be easily modified into various forms like films, fibers, beads, sponges and more complex shapes for orthopedic treatment. The drawbacks of chitosan scaffolds are flexibility and poor mechanical properties, which are inferior to those of normal bone, because of which, they are unable to support load bearingbone implants (15).

It is well known that combination of two materials with different characteristics leads to composites with tailormade properties, so the addition of Hydroxyapatite(HA) to the chitosan scaffold has been shown to increase cell adhesion, cell proliferation, mechanical strength, alkaline phosphatase activity, protein adsorption, type I collagen production as well as expression of other osteogenic differentiation markers $(11,16)$.

Osteoporosis is a disorder that causes a decrease in bone mass that is normally mineralized due to an imbalance between osteoclast and osteoblast activities (17). Osteoporosis is an amorphous process due to the irregular degenerative process, which is difficult to characterize, within a wide range of bone mineralization. Change in bone mineralization is strongly influenced by the nature of atoms capable of performing a substitution to form a composite (18).

Bone crystals are extremely small, with a meanlength of $50 \mathrm{~nm}$ (within range of 20-150 nm), an average width of $25 \mathrm{~nm}$ (in range of $10-80 \mathrm{~nm}$ ), and a thickness of only 2-5 nm. Crystal in osteoporotic bone is calcium phosphate hydroxide with chemical formula of $\mathrm{Ca} 10(\mathrm{OH}) 2(\mathrm{PO} 4) 6$. Crystal of normal boneis sodium calcium hydrogen carbonate phosphate hydrate with chemical formula of Ca8H2 (PO4)6H2O-NaHCO3-H2O (19).

Crystal size in osteoporotic bone is smaller than that in normal bone. This is presumably due to the irregular atomic arrangement in the osteoporotic bone crystal in which crystallographic parameters in osteoporotic bone are not known, while in normal bone, crystallographic parameters are known well in the form of hexagonal crystal system. Moreover, osteoporotic bone crystal showed a lower crystallinity than normal bone, and the atom density of osteoporotic bone crystals is smaller than that of normal bone (19).

The mechanism of glucocorticoid effect on bone metabolism is rather complex, due to its role in arresting wound healing. Glucocorticoids modify osteoblastic cell differentiation, their number and function, thus inhibiting bone formation. Inhibition of bone formation is simultaneously followed by bone resorption and subsequent bone loss (20).

After surgical procedure of tooth extraction, a coagulum fulfils the alveolar socket and a process of wound healing occurs. The healing in osteoporosis never allows restoration to original condition of the alveolar bone ridge, resulting in decreased bone volume and physiological resorption which is a significant clinical problem (21).

Experimental models of osteoporosis in rabbits are useful to investigate anabolic agents because rabbits have an active Haversian remodeling and achieve skeletal maturity quickly (22).

The aim of the study was to assess the efficacy of nanohydroxyapatite/chitosan composite scaffolds in healing alveolar bone defects in rabbits with induced osteoporosis.

\section{MATERIALS AND METHODS}

Experimental animals

Twenty adult female New Zealand white rabbits of 6 months old (3.5-4.5 kg in weight) were used in this study. Animals were obtained from the animal house of Medical Research Institute, Alexandria University. They were kept under the same nutritional and environmental conditions in the experimental animal house (23).

Rabbits were divided randomly by computer assisted software into 2 equal groups: Group A: osteoporosis, with no CS/nHA Scaffold.

Group B: osteoporosis with CS/nHA Scaffold.

Each rabbit in this study followed these criteria (23); Inclusion criteria: The rabbits in this study were matched with age, weight, sex, unified type of diet, and housing. Exclusion criteria: Specifically excluded rabbits that were included in any previous experimental study or presence of any illness or wound.

In order to minimize risk of confounding, random allocation must be truly done, by using a computergenerated random sequence of numbers to assign treatment status.The participant and all personnel who perform the study were unaware of treatment assignment (24).

\section{Materials}

\section{Chitosan-nHA Scaffold}

Chitosan-nHA scaffold was prepared \& purchased from nanotech company (Nanotech company, 6th of October city).

\section{Methods}

1-Induction of osteoporosis (25)

All rabbits in group A\& B were weighted to enable weight related dose adjustments. Rabbits received methylprednisolone sodium succinate (EPICIO company, Nasr city, Egypt), which was injected intramuscularly at a dosage of $2 \mathrm{mg} / \mathrm{kg} /$ day for 4 consecutive weeks. At the end of 4 weeks all rabbits were prepared for surgical extraction of mandibular right first premolar.

\section{2-Surgical extraction (26)}

\section{Anaesthesia:}

The rabbits were anesthetized with general anesthesia using mixture of $13 \mathrm{mg} / \mathrm{kg}$ of $2 \%$ xylazine hydrochloride and 33 $\mathrm{mg} / \mathrm{kg}$ of ketamine base. The mandibular right first premolar was extracted atraumatically.

Application of CS/nHA scaffold:

Following the extraction, in group A the sockets were left to heal spontaneously without application of any material, while in group B the sockets were loaded with CS/nHA. 
The soft tissues in both groups were sutured by 4-0 Vicryl sutures using a cross-mattress technique to achieve site stability.

After the surgery 5 animals from each group were sacrificed after 2 , and 4 weeks. The mandibles were dissected out and the right halves were separated and fixed in $4 \%$ formaldehyde with $1 \%$ glutaraldehyde [4F1G] for the Scanning Electron Microscopic examinations and Energy Dispersed x-ray analysis [EDXA].

\section{Characterization of the scaffold: (Fig 1)}

The morphology and size of the pores of the scaffold were examined with scanning electron microscopy (SEM) (Faculty of Science, Alexandria University). The scaffold was cut by a razor blade and mounted on aluminum stubs with conductive paint and then sputter-coated with gold for examination. The structural morphology of CS/nHA scaffold showed an internal microstructure with spherical, interconnected pores [range from 20-100 $\mu \mathrm{m}$ ] and dense struts composed of nHA particles embedded within a high density.

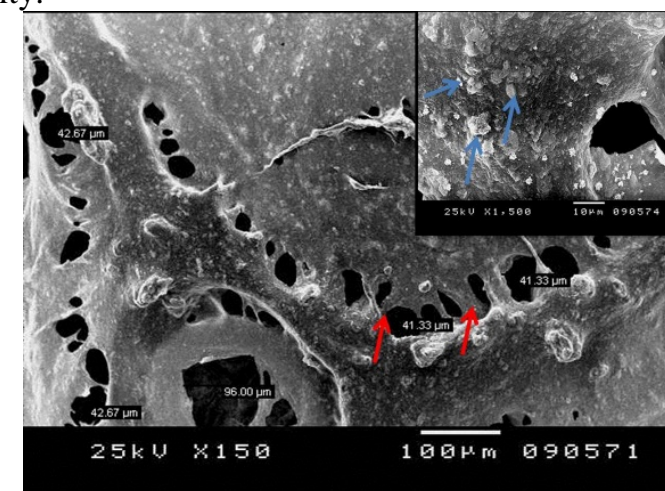

Figure (1): Scanning Electron Micrograph [SEM] of CS/nHA scaffold showing spherical, interconnected pores and dense struts (red arrows) (x150). Inset: showing higher magnification nHA particles (blue arrows) attached on the surface of the scaffold (x1500).

\section{Characterization of the nanohydroxyapatite particles(nHA): (Fig 2)}

The size and shape of nHA were determined using Transmission Electron Microscope (TEM) (Faculty of Science, Alexandria University), which was rod or nanoneedle-like particles, with average particle size range of $20-50 \mathrm{~nm}$.

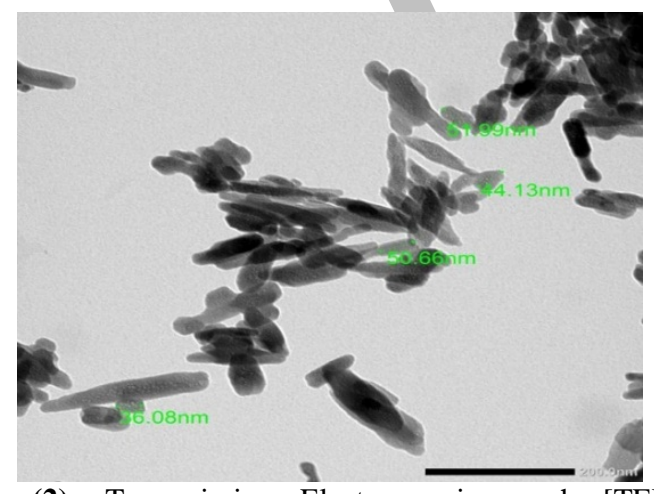

Figure (2): Transmission Electron micrograph [TEM] of nanohydroxyapatite (nHA) suspension showing that most of the nanoparticles are rod or needle like with average particle size ranging from $20-50 \mathrm{~nm}$. (x2000).

\section{-Ultrastructural evaluation of bone regeneration: (27)}

Bone regeneration was evaluated by SEM to assess the newly formed bone trabeculae in all groups. Samples from osteoporotic and CS/nHA group were collected 2 and 4 weeks after extraction, and were fixed in 4F1G. The socket areas were split longitudinally in a buccolingual direction at the mandibular right first premolar region, using a straight head hand piece with a double abrasive disc. We make a groove at the buccal, lingual plate of bone, and basal bone at the center of the socket, and then we split the two halves away from each other. Each half was immersed in phosphate buffers, dehydrated through a 30-100 \% alcohol series at room temperature $\left(24^{\circ} \mathrm{C}\right)$, dried at the critical point, coated with gold in vacuum, and observed by SEM.

-Energy Dispersive x-ray Analysis (EDXA) (Faculty of Science, Alexandria University) (27): EDXA is analytical technique used for the elemental analysis of a sample. It was used to compare the different percentages of bone calcium and phosphate content of the newly formed bone in the osteoporotic and CS/nHA group.

\section{-Ethical Considerations:}

The study was performed after gaining the approval of the Research Ethics Committee, Faculty of Dentistry, Alexandria University.

-Statistical analysis: (28)

Data of EDXA analysis were fed to the computer and analyzed using IBM SPSS software package version 20.0. Quantitative data were described using range (minimum and maximum), mean, standard and median. The distributions of quantitative variables were tested using Ftest (ANOVA). Significance of the obtained results was judged at the $5 \%$ level.

\section{RESULTS}

The results obtained from this study included the followings:

1. Scanning Electron Microscope (SEM)

2. Energy Dispersive X-ray Analysis (EDXA)

1. Scanning Electron Microscope (SEM):

\section{2 weeks interval:}

Group A (osteoporosis): (Fig 3)

Scanning Electron Microscopic examination of this group revealed extensive, irregular, resorptive (pitted) surface and very thin bony spicules with wide marrow spaces. The inset in this micrograph showed higher magnification of osteocytes with irregular lacunae space and large amount of unmineralized collagen (blue arrows).

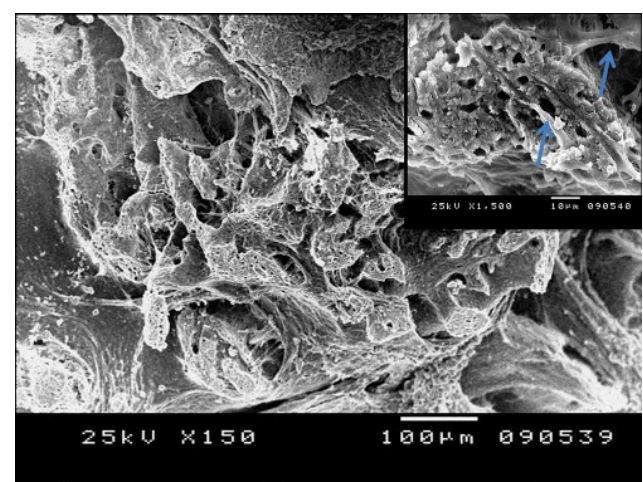

Figure (3): SEM micrograph of Group A (osteoporosis) after (2 weeks) showing irregular, resorptive (pitted) surface and very thin bony spicules (x150). Inset: higher magnification showing 
osteocytes with irregular lacunae and large amount of unmineralized collagen (blue arrows) (x1500).

\section{Group B (osteoporosis, CS/nHA Scaffold): (Fig 4)}

Scanning Electron Microscopic examination of this group showed more regular bone formation with large uniform woven bony spicules and relatively narrow bone marrow spaces. The inset is higher magnification of this micrograph that showed osteocyte, with uniform lacunae (blue arrows) and regular surface of the newly formed bone represented more bone formation.

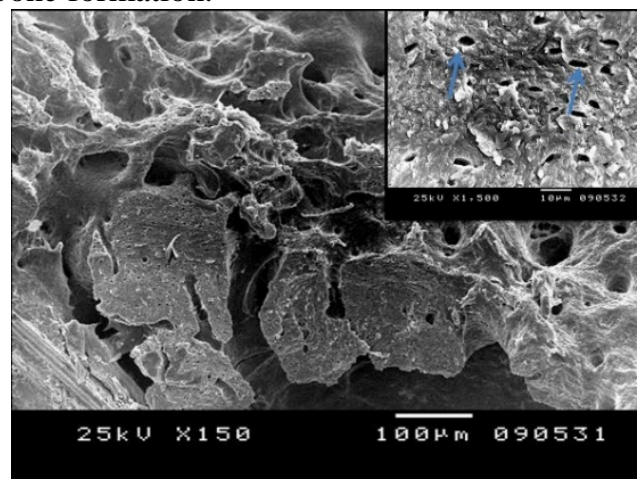

Figure (4): SEM micrograph of Group B (osteoporosis, CS/nHA Scaffold) after (2 weeks) showing internal regular bone formation with smooth woven bony spicules and relatively narrow bone marrow spaces (x150). Inset: higher magnification showing osteocyte with uniform lacunae (blue arrows) and regular surface of the newly formed bone(x1500).

4 weeks interval:

\section{Group A (osteoporosis): (Fig 5)}

In the osteoporotic group, the Scanning Electron Microscopic examination calcifying bone matrix were observed at newly formed bone after 4 weeks with resorptive (pitted) surface, it represent a slow rate of bone formation. The inset is higher magnification of this micrograph that showed unmineralized collagen (blue arrows).

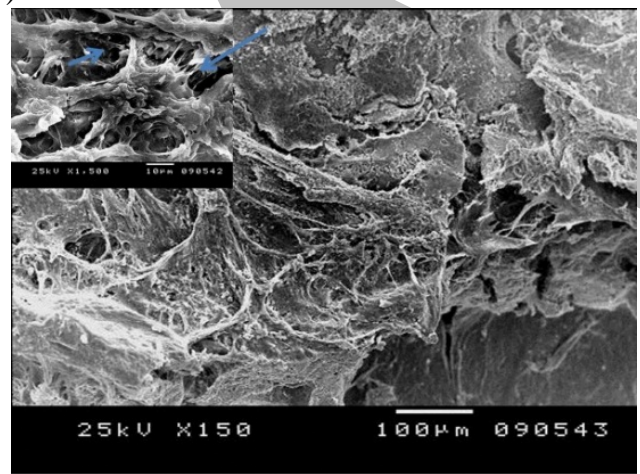

Figure (5): SEM micrograph of Group A (osteoporosis) after (4 weeks) showing irregular, resorptive (pitted) surface, and less calcifying bone matrix (x150). Inset: higher magnification of this micrograph showing less mineralized collagen (blue arrows) (x1500).

\section{Group B (osteoporosis,CS/nHA Scaffold): (Fig 6)}

At 4 weeks, Scanning Electron Microscopic examination of this group revealed a large amount of newly formed bone, as well as abundant calcifying matrix. The inset is higher magnification of this micrograph that showed osteocytes with uniform lacunae (red arrows) and regular surface of the newly formed bone with calcifying globules represented bone mineralization (blue arrows).

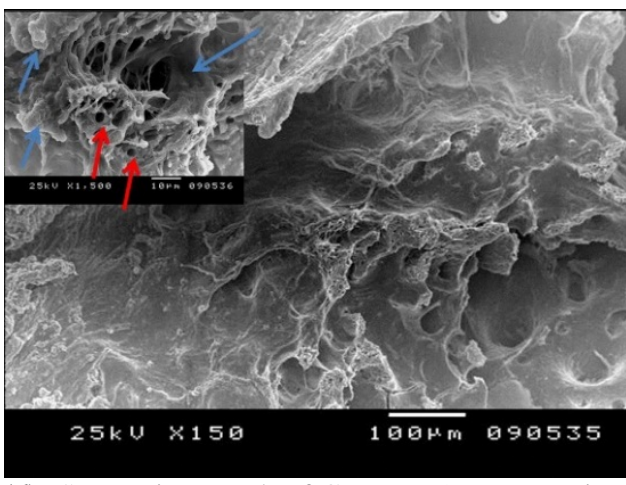

Figure (6): SEM micrograph of Group B (osteoporosis, CS/nHA Scaffold) after (4 weeks) showing rapid bone formation with smooth surface (x150). Inset: higher magnification of this micrograph showing osteocytes with uniform lacunae (red arrows) and regular surface of the newly formed bone with calcifying globules represented bone mineralization (blue arrows) (x1500).

2. Energy Dispersive x-ray Analysis (EDXA): (Table 1\& 2) Table (1) shows comparison between the two groups after the first two weeks, there was a significantly decrease in the percentage of $\mathrm{Ca}$ in osteoporotic group (A) than CS/nHA scaffold group (B), with mean values were $47.9 \pm 5.3$ and $64.2 \pm 2.4$ respectively and significantly increases in the percentage of $\mathrm{P}$ with mean values were $52.1 \pm$ 5.3and35.8 \pm 2.4 respectively, with $P$ value $=0.001$.

Table (2) show comparison between the two groups after four weeks, the results of Ca increased significantly more in CS/nHA scaffold group (B) than the osteoporotic group (A), with mean values were $774.0 \pm 3.4$, and $58.6 \pm$ 2.0, respectively. While the percentage of $\mathrm{P}$ decreased more in CS/nHA scaffold group (B) than the osteoporotic group (A), with mean values were $26.0 \pm 3.4$, and 41.4 \pm 2.0 , respectively, with $\mathrm{P}$ value $<0.001$.

Table (1): Comparison between the two studied groups according to $\mathrm{P}$ and $\mathrm{Ca}$ after two weeks

\begin{tabular}{|c|c|c|c|c|c|}
\hline & & $\begin{array}{l}\text { Group A } \\
(n=4)\end{array}$ & $\begin{array}{l}\text { Group B } \\
(n=4)\end{array}$ & $\mathbf{t}$ & p \\
\hline \multicolumn{6}{|c|}{$\mathbf{P}$} \\
\hline & Mean \pm SD. & $52.1 \pm 5.3$ & $35.8 \pm 2.4$ & \multirow[b]{2}{*}{$5.568^{*}$} & \multirow[b]{2}{*}{$0.001^{*}$} \\
\hline & $\begin{array}{l}\text { Median (Min. - } \\
\text { Max.) }\end{array}$ & $\begin{array}{l}52.9(45.2- \\
57.5)\end{array}$ & $\begin{array}{l}35.6(33.2- \\
38.9)\end{array}$ & & \\
\hline \multicolumn{6}{|c|}{ Ca } \\
\hline & Mean \pm SD & $47.9 \pm 5.3$ & $64.2 \pm 2.4$ & \multirow[b]{2}{*}{$5.568^{*}$} & \multirow[b]{2}{*}{$0.001^{*}$} \\
\hline & $\begin{array}{l}\text { Median (Min. - } \\
\text { Max.) }\end{array}$ & $\begin{array}{l}47.2(42.5- \\
54.8)\end{array}$ & $\begin{array}{l}64.5(61.1- \\
66.8)\end{array}$ & & \\
\hline
\end{tabular}

\section{t: Student t-test}

$\mathrm{p}$ : p value for comparing between the studied groups

*: Statistically significant at $\mathrm{p} \leq 0.05$

Table (2): Comparison between the two studied groups according to $\mathrm{P}$ and $\mathrm{Ca}$ after four weeks

\begin{tabular}{|l|l|l|l|l|}
\hline & $\begin{array}{l}\text { Group A } \\
(\mathbf{n = 4 )}\end{array}$ & $\begin{array}{l}\text { Group B } \\
(\mathbf{n = 4 )}\end{array}$ & $\mathbf{t}$ & $\mathbf{p}$ \\
\hline P & & & & \\
\hline Mean \pm SD. & $41.4 \pm 2.0$ & $26.0 \pm 3.4$ & $7.803^{*}$ & $<0.001$ \\
\hline $\begin{array}{l}\text { Median (Min. - } \\
\text { Max.) }\end{array}$ & $\begin{array}{l}40.9(39.6- \\
44.3)\end{array}$ & $\begin{array}{l}25.4(22.6- \\
30.6)\end{array}$ & & \\
\hline Ca & & & & \\
\hline Mean \pm SD. & $58.6 \pm 2.0$ & $74.0 \pm 3.4$ & $7.803^{*}$ & $<0.001$ \\
\hline $\begin{array}{l}\text { Median (Min. - } \\
\text { Max.) }\end{array}$ & $\begin{array}{l}59.1(55.7- \\
60.4)\end{array}$ & $\begin{array}{l}74.6(69.4- \\
77.4)\end{array}$ & & \\
\hline
\end{tabular}

t: Student t-test

$\mathrm{p}$ : $\mathrm{p}$ value for comparing between the studied groups

$*$ : Statistically significant at $p \leq 0.05$ 


\section{DISCUSSION}

Osteoporosis is characterized by reduced bone mass and disruption of bone architecture, resulting in increased risks of fragility fractures which represent the main clinical consequence of the disease (29). It is classified into primary and secondary types. Primary osteoporosis refers to postmenopausal osteoporosis and to age-related osteoporosis. In contrast, secondary osteoporosis refers to bone disorders that are secondary complications of various other medical conditions, it has been established that glucocorticoids are the most commonly implicated drug that accompanied with significant bone loss (30).

Glucocorticoids cause general skeletal resorption as well as focal damage in idiosyncratic areas of the skeleton. Because glucocorticoids affect osteoblastic and osteoclastic differentiation and activity, determining the mechanisms that are involved has been complex. The conditions used in this study, including the 30-day experimental time, allowed demonstration of changes including significant bone loss but without advanced lesions, at a controlled glucocorticoid dose (31). For this reason, we used experimental dose of methylprednisolone acetate $2 \mathrm{mg} / \mathrm{kg} / \mathrm{day}$. No fractures or osteonecrosis were apparent in this group, and gave consistent and measurable bone changes. This is in accordance with a study done by Eberhardt et al (32).

Tooth extraction was once described as a tissue amputation that may lead to functional, psychological, postural and local changes. In modern dentistry, the healing process of the socket following tooth extraction has become an important topic of research, study and discussion (32).

In the present study, rabbit was chosen to investigate socket healing. Using rabbit as an experimental animal has many advantages including its availability, economic expenses, short life span and healing short duration, also females are more prone for osteoporosis than males, so we used females in our study (33). The right first mandibular premolars were extracted to be used as a model to study socket healing.

In the current study, the healing of extraction socket was evaluated at two different intervals 2 and 4 weeks respectively, Fisher et al (34) used approximately the same intervals.

To the best of our knowledge, the review of literature is lacking articles dealing with assessment of the effect of $\mathrm{CS} / \mathrm{nHA}$ composite on the healing of extraction socket in rabbit with induced osteoporosis. So the aim of the present study was directed to reveal the result of such effect.

The current study demonstrates that the extracted socket defects of the rabbits with induced osteoporosis can be repaired by nanohydroxyapatite/CS composite scaffold within four weeks. Nanohydroxyapatite belongs to ceramicbased bone graft substitutes. Besides nanohydroxyapatite, the main materials of this category include tri-calcium phosphate (TCP), bioactive glass ceramics (Bio glass), calcium phosphate cement (CPC), and calcium sulfate (35).

It is well known that the high proportion of HA and uniform distribution of nHA granules in the CS matrix enable the scaffolding material to possess good biocompatibility, high bioactivity and enough mechanical strength, in addition the higher content of $\mathrm{HA}$ in the composite, the better bioactivity can be achieved by the material(6).Therefore, to enhance the bioactivity of the material and to better mimic the hybrid composition of natural bone and enhance the mechanical strength of the scaffold, we chose the CS /nHA scaffold.

Chitosan [CS] is a natural polysaccharide and is considered the largest biomaterial after cellulose in terms of utilization and distribution. Chitosan has attracted attention because of its biological properties and effective uses in the medical field, food industries, and agricultural sector. It shows a variety of biological activities such as activation of immune response, cholesterol lowering activity, and antihypertension activity, inhibition of growth of microorganisms and pain alleviation and promotion of hemostasis and epidermal cell growth (14).

The beginning of bone formation and bone cell proliferation is a very complex process. Some research has found that three-dimensional pore structures could facilitate cell adhesion, differentiation, and proliferation, and help with fibrovascular and nerve colonization, so that the new bone "crawls" into the material to achieve early osteoinduction. The surface of a nano-material is significantly larger than a micron-material, and the numbers of atoms are significantly increased on the surface. Thereby, owing to their greater surface area, the nano-sized particles are more biologically active compared with micron-sized particles (6). The previous studies have shown that micronHA only had osteoconduction without osteoinduction, yet nano-HA had both $(6,12,13,15)$.

After Characterization of the scaffold, we found that nHA/CS nanocomposite showed internal microstructure with spherical, regular interconnected pores [range from 20$100 \mu \mathrm{m}$ ] and dense struts composed of nHA particles embedded within a high density, this is in accordance with study done by Woodard et al (36) Who stated that the Scaffold architecture plays an important role in determining the rate and degree of bone ingrowth. Macroporosity (pores $>50 \mu \mathrm{m}$ ) is thought to contribute to osteogenesis by facilitating cell and ion transport and provide spacing for the vasculature required to nourish new bone. Studies suggest that microporosity (pores $<20 \mu \mathrm{m}$ ) improves bone growth into scaffolds by increasing surface area for protein adsorption, and providing attachment points for osteoblasts $(36,38)$, that all was approved in our study.

After characterization of the nanohydroxyapatite particles (nHA)particles, it showed that the shape of nHA with rod or nanoneedle-like particles with average particle size was observed to be in the range of $20-50 \mathrm{~nm}$, the same results were conducted by Uskoković et al (37).

The SEM results of the present work demonstrated that at the end of the second week after extraction, the socket of nHA/CS group have more regular and rapid woven bone formation with large number of osteocytes inside its lacunae indicating active bone formation than the osteoporotic group.

Kong etal (38) showed the reason for increased bone formation in his study, where the compatibility between bone forming cells and the chitosan/nano-HA composite scaffolds was evaluated by MC3T3-E1 [a preosteoblast cell line derived from newborn mouse calvaria], It can be seen that the cells on the apatite layer formed on composite scaffolds were distributed in clusters having more cells than those formed on chitosan scaffolds alone, this was often related to well-spreaded cell adhesion, attaching its filapodia to the hydroxyapatite nanoparticles. Also the Alkaline phosphatase (ALP) activity of cells on composite scaffolds was much higher than that of cells on chitosan 
scaffolds. The ALP activity assessment indicated that cells on the composite scaffolds showed higher differentiation level than chitosan scaffolds (38).

The better performance of nanocomposite could be explained from three aspects as follow. First, favorable osteoconductivity. The three-dimensional CS /nHA nanocomposite scaffolds are similar to bone apatite in size, phase composition and crystal structure, with the pore diameter ranging from 20 to100 nm. They serve as a template to guide bone tissue regeneration, while it gradually degrades and replaces by newly formed bone tissue. The porosity and pore size play a critical role in the growth of cells (36).

Second, good osteogenesis in CS/nHA scaffolds were characterized by a relatively rough surface and approximately 20 times greater area/unit mass than CS scaffold, which indicated increase adsorption of fibronectin and improved cell attachment. The biological response of pre-osteoblasts on nanocomposite scaffolds was superior in terms of improved cell attachment, higher proliferation, and well-spread morphology in relation to CS scaffold, as reported by Thein-Han et al (10).

Third, favorable osteoinductivity. The nanocomposite is able to support mitogenesis of undifferentiated cells and help them to differentiate into bone $(36,10)$.

Four weeks after the operation, the regular trabeculae were observed in bone defect, because of its favorable osteoconductivity properties, that was previously reported for CS/nHA composite (36).

At the second week after extraction, the socket of osteoporotic group has irregular, resorptive (pitted) surface and very thin bony spicules were observed at the surface of newly formed bone. this fact is explained by Zhao et al (39) study as osteoporotic bone originated osteoblast showed significantly lower Alkaline Phosphatase [ALP] activity, matrix mineralization ability and gene expressions, including ALP, Osteocalcin [OCN], Bone Sialoprotein [BSP] and Osterix [OSX] (39).

At the end of four week after extraction, the socket of osteoporotic group were observed at the surface of newly formed bone, with resorptive (pitted) surface, and it showed a slow rate of bone formation.

The explanation of both intervals in our study was done by Eberhardt etal (31) who stated that glucocorticoids cause damage bone by a mechanism that includes osteocyte and osteoblast death, most likely by apoptosis of contiguous groups of cells in osteons. Apoptosis of osteocytes is considered as the third cause of osteoporosis that appears to be responsible for the decreased bone formation. In addition, this study demonstrated that an increase in apoptotic osteocytes that may contribute to bone fragility independent of changes in bone mass.

In addition, Glucocorticoids function in normal bone turnover, thus probably reflects extreme stimulation of a physiologically important receptor, as osteoblast expression of Receptor activator of nuclear factor $\kappa \mathrm{B}$ ligand [RANK ligand], that is a key pathway in osteoclast development, also it has also been noted that glucocorticoids stimulate in bone the formation of cAMP, the principal second messenger mediating parathyroid hormone [PTH] activity (39).

The EDXA analysis confirmed the results of the SEM, where after the first healing period; it has been revealed that the percentage of the Calcium of the newly formed bone was lowest in osteoporosis group and highest in CS/nHA Scaffold group. The difference in percentage of newly formed bone between osteoporosis group and CS/nHA Scaffold group was statistically significant.

Calcium is beneficial for cell proliferation. While phosphate ions release from nHA/CS composites can absorb calcium from ambient environment. The mechanism underlying the upregulated cellular functions observed and how cells would respond to nanohydroxyapatite particles (nHA) at subcellular level was reported by Zhao et al (39). Homeostasis of intracellular calcium plays a vital role in the regulation of cell proliferation and growth, especially in osteoblastic cells. A rise in intracellular calcium level in osteoblastic cell line was found to be a necessary process in both Bone morphogenetic proteins-2 [BMP-2] induced differentiation, as well as microdamaged bone repair especially in osteoporosis.

Finally, we recommended the use of chitosan/nHA composite scaffold in osteoporotic induced bone regeneration, as the satisfactory repair rate of the extraction socket suggested the determining role of chitosan/nHA scaffold in facilitating new bone regeneration.

\section{CONCLUSION}

The finding of this study proved that Osteoporosis is a disease that dramatically affects bone healing \& declared that chitosan/nHAscaffold improved bone regeneration thus it can be used in case of osteoporosis to counter act its effect.

\section{CONFLICT OF INTEREST}

The authors declare that they have no conflicts of interest.

\section{ACKNOWLEDGMENTS:}

I would like to thank prof.Dr Afaf El-Sawa, prof.Dr. SaharShafik, and Dr. Sara Ashraf for providing support in this study.

\section{REFERENCES}

1. Porter AL, Youtie J, Shapira P, Schoeneck DJ. Refining search terms for nanotechnology. J Nanopart Res.2008;10:715-28.

2. Dvir T, Timko BP, Brigham MD, Naik SR, Karajanagi SS, Levy O, et al. Nanowired three-dimensional cardiac patches. Nat Nanotechnol.2011;6:720-5.

3. Taylor E, Webster TJ. Reducing infections through nanotechnology and nanoparticles. Int $\mathrm{J}$ Nanomedicine. 2011;6:1463-73.

4. Paul W, Sharma CP. Nanoceramic Matrices: Biomedical Applications. Am J Biochem Biotechnol. 2006;41-48.

5. McMahon RE, Wang L, Skoracki R, Mathur AB. Development of nanomaterials for bone repair and regeneration. J Biomed Mater Res B Appl Biomater.2013;101:387-97.

6. Groeneveld EH, van den Bergh JP, Holzmann P, ten Bruggenkate CM, Tuinzing DB, Burger EH. Mineralization processes in demineralized bone matrix grafts in human maxillary sinus floor elevations. J BiomedMater Res.1999;48:393-402.

7. Bezzi G, Celotti G, Landi E, La Torretta T, SopyanI, Tampieri A. A novel sol-gel technique for hydroxyapatite preparation. Mater Chem Phys. 2003;78: 816-24. 
8. Koutsopoulos S. Synthesis and characterization of hydroxyapatite crystals: a review study on the analytical methods. J Biomed Mater Res.2002;600-12.

9. Lee JS, Baek SD, Venkatesan J, Bhatnaga I, Chang HK, $\mathrm{Kim} \mathrm{HT}$, et al. In vivo study of chitosan-natural nano hydroxyapatite scaffolds for bone tissue regeneration. Int $\mathrm{J}$ Biol Macromol.2014;67:360-6.

10. Thein-Han WW, Misra RD. Biomimetic chitosannanohydroxyapatite composite scaffolds for bone tissue engineering. Acta Biomater. 2009;5:1182-97.

11. Konishi S, Nakamura H, Seki M, Nagayama R, Yamano Y. Hydroxyapatite granule graft combined with recombinant human bone morphogenic protein-2 for solidlumbar fusion. J Spinal Disord Tech.2002;15:237-44.

12. Chen F, Wang ZC, Lin CJ. Preparation and characterization of nano-sized hydroxyapatiteparticles and hydroxyapatite/chitosan nano-composite for usein biomedical materials. Mater Lett.2002;57:858-61.

13. Chen F, Lam WM, Lin CJ, Qiu GX, Wu ZH, Luk KD, et al. Biocompatibility of electrophoretical deposition of nanostructured hydroxyapatite coating on roughen titanium surface: in vitro evaluation using mesenchymal stem cells. J Biomed Mater Res B Appl Biomater.2007;82:183-91.

14. Mincea M, Negrulescu A, Ostafe V. Preparation, modification, and applications of chitin nanowhiskers: a review. Rev Adv Mater Sci.2012;30:225-42

15. Pallela R, Venkatesan J, Janapala VR, Kim SK. Biophysicochemical evaluation of chitosan-hydroxyapatitemarine sponge collagen composite for bone tissue engineering. J Biomed Mater Res A. 2012;486-95.

16. Huang H, Feng Q, Yu B, Li S. Biomimetic properties of an injectable chitosan/nano-hydroxyapatite/collagen composite. Mater Sci Eng C.2011;31:683-7.

17. Duncan EL, Brown MA. Genetic studies in osteoporosisthe end of the beginning. Arthritis Res Ther.2008;10:214.

18. Huang QY, Kung AWC. Genetics of osteoporosis. Mol Genet Metab Rep. 2006;88:295-306.

19. Noor Z, Substitution and incorporation of atomic minerals,hydroxyapatite crystale and microstructure of osteoporosis bone. Ph.D. Thesis. Faculty of Medicine, Brawijaya University, East Java Malang, Indonesia. 2011.

20. Canalis E. Mechanism of glucocorticoid-induced osteoporosis. Curr Opin Rheumatol. 2003;15: 454-7.

21. Karring T, Lang NP, Lindhe J. Clinical Periodontology and Implant Dentistry. $4^{\text {th }}$ ed. Oxford: Blackwell Publishing; 2003.

22. Baofeng L, Zhi Y, Bei C, Guolin M, Qingshui Y, Jian L. Characterization of a rabbit osteoporosis model induced by ovariectomy and glucocorticoid. Acta Orthop.2010;81:396401.

23. Navia JM, University of Alabama Press. Animal models in dental research. Int J Oral Maxillofac Surg. 1977;17:82-83.

24. Muhlhausler BS, Bloomfield FH, Gillman MW. Whole animal experiments should be more like human randomized controlled trials. PLoS Biol.2013;11: e1001481.
25. Lin T, Liu J, Yang S, Liu X, Feng X, Fu D. Relation between the development of osteoporosis and osteonecrosis following glucocorticoid in a rabbit model. Indian J Orthop.2016;50:406-13.

26. Kotsakis G, Chrepa V, Marcou N, Prasad H, Hinrichs J. Flapless alveolar ridge preservation utilizing the "socketplug" technique: clinical technique and review of the literature. J Oral Implantol 2012.

27. Kotz S, Balakrishnan N, Read CB, Vidakovic B. Encyclopedia of statistical sciences. $2^{\text {nd }}$ ed. Hoboken, NJ: Wiley-Interscience; 2006.

28. Svedbom A. Hernlund E. Ivergård M. Epidemiology and Economic Burden of Osteoporosis in Austria; A report prepared in collaboration with the International Osteoporosis Foundation (IOF) and the European Federation of Pharmaceutical Industry Associations (EFPIA). Arch Osteoporos. 2013;8:137.

29. Weinstein RS. Clinical practice. Glucocorticoid-induced bone disease. N Engl J Med. 2011;365:62.

30. Eberhardt AW, Yeager-Jones A, Blair HC. Regional trabecular bone matrix degeneration and osteocyte death in femora of glucocorticoid-treated rabbits. Endocrinology.2001;142:1333-40.

31. Araújo MG, Silva CO, Misawa M, Sukekava F. Alveolar socket healing: what can we learn? Periodontology 2000. 2015;68:122-34.

32. Nicklas W, Baneux P, Boot R, Decelle $T$, Deeny AA, Fumanelli $M$, et al. Recommendations for the health monitoring of rodent and rabbit colonies in breeding and experimental units. Lab Anim. 2002;36:20-42.

33. Fisher JP, Lalani Z, Bossano CM, Brey EM, Demian N, Johnston CM, et al. Effect of biomaterial properties on bone healing in a rabbit tooth extraction socket model. J Biomed Mater Res A.2004;68:428-38.

34. Nandi SK, Roy S, Mukherjee P, Kundu B, De DK, Basu D. Orthopaedic applications of bone graft \& graft substitutes: a review. Indian J Med Res.2010;132:15-30.

35. Woodard JR, Hilldore AJ, Lan SK, Park CJ, Morgan AW, Eurell JA. et al. The mechanical properties and osteoconductivity of hydroxyapatite bone scaffolds with multi-scale porosity. Biomaterials.2007;28:45-54.

36. Uskoković V, Uskoković DP. Nanosized hydroxyapatite and other calcium phosphates: Chemistry of formation and application as drug and gene delivery agents. J Biomed Mater Res B Appl Biomater.2011;96:152-91.

37. Kong L, Gao Y, Guangyuan LU, Gong Y, Zhao N, Zhang $X$. A study on the bioactivity of chitosan/nanohydroxyapatite composite scaffolds for bone tissue engineering. Eur Polym J 2006;42:3171-9.

38. Zhao R, Xie P, Zhang K, Tang Z, Chen X, Zhu X, et al. Selective effect of hydroxyapatite nanoparticles on osteoporotic and healthy bone formation correlates with intracellular calcium homeostasis regulation. Acta Biomater.2017;59:338-50. 